\title{
Structure of in vivo protein crystals from viviparous Diploptera punctata
}

Sanchari Baneriee $^{1}$, Nathan P Coussens ${ }^{2}$, François-Xavier Gallat ${ }^{3}$, Nitish Sathyanarayanan ${ }^{1}$, Koichiro J. Yagi ${ }^{4}$, James S. S. Gray ${ }^{5}$, Stephen S. Tobe ${ }^{4}$, Barbara Stay ${ }^{6}$, Leonard M.G. Chavas ${ }^{7}$, S Ramaswamy ${ }^{1}$

${ }^{1}$ Institute Of Stem Cell Biology And Regenerative Medicine, Bangalore, India, ${ }^{2}$ National Center for Advancing Translational Sciences, National Institutes of Health, MD, United States, ${ }^{3}$ Structural Biology Research Center, High Energy Accelerator Research Organization, Tsukuba-city, Japan, ${ }^{4}$ Department of Cell and Systems Biology, University of Toronto, Toronto, Canada,

${ }^{5}$ Department of Biochemistry, Carver College of Medicine, University of Iowa, Iowa City, United States, ${ }^{6}$ Department of Biology, University of Iowa, Iowa City, United States, ${ }^{7}$ Synchrotron SOLEIL, Gif-sur-Yvette Cedex, France

E-mail: sancharib@instem.res.in

In many organisms, several proteins are known to crystallize in vivo and have been proposed to be under positive selection pressure for crystallizing inside the cells. Common examples of such proteins that have been linked to biological functions include insulin secretion, sorting of secretory proteins in the Golgi apparatus, pathogenesis of Bacillus thuringiensis, as storage mechanisms for infectious viruses and developmental proteins in seeds and eggs. While natural crystallization of proteins inside cells has been identified in different kingdoms of life, the structures of only a few crystals have been determined although none at atomic resolution. We have determined the first atomic resolution (1.2 $\AA$ ) structure from in vivo grown protein crystals solved by sulfur single-wavelength anomalous dispersion phasing using lower energy $\mathrm{X}$-rays. These are crystals of lipocalin-like milk protein from the cockroach, Diploptera punctata and found to crystallize in vivo in the midgut of milk-drinking cockroach embryos. These crystals serve as calorie intense complete nutrients for the embryo development. Typically, in vitro crystallization requires highly pure and homogeneous proteins. Our structure reveals a motif that is heterogeneous in protein sequence, glycosylation as well as the bound lipid and serves as a complete food for cockroaches. In vitro expression and secretion of the milk protein in yeast is in progress. Development of the recombinant form of this protein will help in making many products like nutritional supplements, fortified food, poultry feed, hydrophobic drug/vitamin carrier etc.

[1] Banerjee, S et al., (2016). IUCr], 3, 282-293

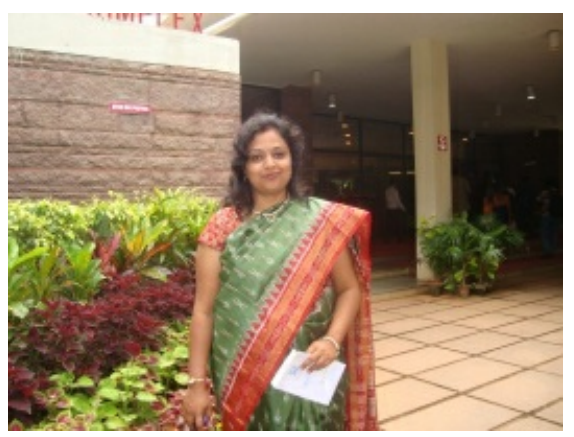

Keywords: sulfur-SAD, viviparity in cockroach, protein heterogeneity. 